\title{
Mapping of optimum placement of distributed generation in meshed power networks with appropriate technologies
}

\begin{abstract}
Purpose: This study aims to propose a sensitivity-based methodology for the optimum accommodation of distributed generation (DG) units in meshed power networks with appropriate technologies. The effect of load variation is incorporated into the proposed methodology to identify the most trusted locations for DG placement. Design/methodology/approach: The effectiveness of minimizing active power losses is considered a key criterion. A priority list comprising both sensitivity indexes and realistic indicators is deduced to rank the optimum sites for the placement of DG units. A sorting index for distinguishing the suitable DG type(s) for each candidate location is organized. Three common DG types are considered in this work. The modified IEEE 30-bus meshed system is chosen to perform the proposed methodology. Findings: Results demonstrate that the obtained priority index can be used to achieve the best real loss minimization rates. Numerous load buses can be safely excluded as candidate locations using the proposed approach. Consequently, the methodology can minimize the computational process of diagnosing the optimum sites for DG accommodation. Originality/value: The findings determine that instead of installing many DG units at various locations with one DG type, a few certain load buses can be used to accommodate more than one DG type and significantly reduce losses.
\end{abstract}

Keyword: Distributed generation; Sensitivity analysis; Power loss minimization; Meshed power networks 